\title{
Proposta de plano de cuidados para pessoas em situação de rua
}

\author{
Care plan proposal for person in street situation
}

\author{
Aline Moreira Ximenes ${ }^{1}$ \\ Orcid: http://orcid.org/0000-0002-1674-3357
}

Iara Fonteles Muniz ${ }^{3}$

Orcid: http://orcid.org/0000-0002-5444-8340

Joselany Áfio Caetano 5

Orcid: http://orcid.org/0000-0002-0807-056X

\author{
Maria Girlane Sousa Albuquerque Brandão ${ }^{2}$ \\ Orcid: http://orcid.org/0000-0002-9925-4750
}

\author{
Caroline Ponte Aragão 4 \\ Orcid: https://orcid.org/0000-0001-5447-302 \\ Lívia Moreira Barros ${ }^{6}$ \\ Orcid: http://orcid.org/0000-0002-0174-2255
}

\begin{abstract}
Resumo
Introdução: Para uma assistência qualificada à Pessoa em Situação de Rua, é necessário atendimento multidisciplinar com individualização do cuidado. Desse modo, a enfermagem contribui no desenvolvimento de intervenções organizadas e pautadas em abordagem holística do paciente. Objetivo: Propor diagnósticos, resultados e intervenções de enfermagem para Pessoas em Situação de Rua com base em suas atividades de vida diária. Materiais e Métodos: Estudo transversal, de abordagem quantitativa, realizado em Centro de Referência Especializado para Pessoas em Situação de Rua em Sobral, Ceará, Brasil. A amostra contou com 52 participantes. A coleta dos dados ocorreu por entrevista individual, no período de abril a junho de 2017, norteada por questionário estruturado baseado no Modelo de Atividades de Vida Diária. As respostas dos pacientes foram tabuladas, sendo incluídos no plano de cuidados os Diagnósticos de Enfermagem que obtiveram uma frequência maior que $40 \%$. Resultados: Foram identificados 34 diagnósticos de enfermagem, os quais foram divididos conforme as atividades de vida diária (Manutenção de um ambiente seguro, comunicar, respirar, alimentar, eliminar, trabalho e distração, sexualidade, sono e morte), sendo 16 de risco e 18 reais. Conclusão: O plano de cuidados favorece a assistência de enfermagem com base nos diversos problemas que envolvem o processo saúde-doença da população em situação de rua.
\end{abstract}

Palavras-chave: pessoas em situação de rua; planejamento de assistência ao paciente; atividades cotidianas; processo de enfermagem

\footnotetext{
Abstract

Introduction: For qualified assistance to street people, multidisciplinary care with individualized care is necessary. In this way, nursing contributes to the development of

${ }^{1}$ Universidade Federal do Ceará, Brasil. E-mail: Aline Moreira Ximenes.

${ }^{2}$ Universidade da Integração Internacional da Lusofonia Afro-Brasileira, Ceará, Brasil. E-mail: girlane.albuquerque@yahoo.com.br

${ }_{3}^{3}$ Prefeitura Municipal de Jijoca de Jericoacoara, Brasil. E-mail: iaramunizf@gmail.com

${ }^{4}$ Autônomo. E-mail: carolineponte@gmail.com

${ }^{5}$ Universidade Federal do Ceará, Brasil. E-mail: joselany@ufc.br

${ }^{6}$ Universidade da Integração Internacional da Lusofonia Afro-Brasileira, Ceará, Brasil. E-mail: livia.moreirab@hotmail.com
} 
organized interventions based on a holistic approach to the patient. Objective: Propose nursing diagnoses, results and interventions for People on the Street based on their daily activities. Materials and Methods: Cross-sectional study, with a quantitative approach, carried out in a Specialized Reference Center for Homeless People in Sobral, Ceará, Brazil. The sample had 52 participants. Data collection took place through an individual interview, from April to June 2017, guided by a structured questionnaire based on the Daily Living Activities Model. The patients' responses were tabulated, and the Nursing Diagnoses that obtained a frequency greater than $40 \%$ were included in the care plan. Results: 34 nursing diagnoses were identified, which were divided according to the activities of daily living (maintaining a safe environment, communicating, breathing, eating, eliminating, work and distraction, sexuality, sleep and death), 16 of which are at risk and 18 reais. Conclusion: The care plan favors nursing care based on the various problems that involve the health-disease process of the homeless population.

Keywords: street people; patient care planning; daily activities; nursing process

\section{Introdução}

A População em Situação de Rua (PsR) é considerada grupo populacional vulnerável e heterogêneo, que possui em comum a situação de pobreza, moradia não convencional e sobreposição de vulnerabilidades sociais ${ }^{1}$. As taxas de morbidade e mortalidade de PsR, em comparação com a população em geral, são altas, pois são constantemente acometidas por inúmeras doenças infecciosas e não infecciosas, assim como uso abusivo de álcool e outras drogas ${ }^{2}$.

O contingente populacional de PsR está crescendo vigorosamente em diversos países $^{3}$. Além das vulnerabilidades sociais, essa população possui dificuldades para acessar os serviços de saúde, especialmente a atenção primária, o que a leva, muitas vezes, a agudização de doenças, e promove atendimentos emergenciais em unidades de pronto atendimento ${ }^{4}$.

Para viabilizar o acesso desses indivíduos aos cuidados de saúde, o Ministério da Saúde planejou diversas ações com objetivo de resgatar a cidadania dessa população, dentre essas, destaca-se o consultório na rua, que está vinculado à atenção básica, e apresenta como proposta a prevenção de doenças e a promoção da saúde, como forma de subsidiar melhorias na qualidade de vida das $\operatorname{PsR}^{5}$.

Para uma assistência qualificada é necessário atendimento multidisciplinar à PsR, com individualização do cuidado de acordo com as necessidades de cada um. Desse modo, a enfermagem contribui no desenvolvimento de intervenções organizadas e pautadas em abordagem holística do paciente. A Sistematização de Assistência em Enfermagem (SAE) é uma ferramenta metodológica que envolve levantamento de dados, identificação de diagnósticos, planejamento, implementação de cuidados em saúde e avaliação desse processo $^{6}$.

A SAE é baseada em um sistema de classificação taxonômica que permite a identificação de problemas, o agrupamento e interpretação de dados para solucioná-los. Essas taxonomias beneficiam o planejamento de intervenções de enfermagem e a padronização das terminologias entre a equipe multiprofissional.

Configura-se, ainda, como importante recurso por promover a autonomia dos profissionais da Enfermagem, ofertando-lhes, assim, maior segurança nas ações assistenciais, continuidade no cuidado, e maior proximidade entre pacientes e profissionais, com fortalecimento de vínculos ${ }^{7}$.

Em especial, no contexto da população em situação de rua, os diagnósticos, resultados esperados e intervenções de enfermagem são propostos com base em julgamentos clínicos sobre processos vividos pelos indivíduos e envolvem problemas de saúde reais e potenciais que afetam direta ou 
indiretamente suas atividades de vida diária ${ }^{8}$.

Para esses indivíduos, as atividades de vida diária relacionam-se diretamente com bem-estar e sobrevivência. Desse modo, torna-se relevante associar o uso de metodologias próprias da enfermagem com as atividades de vida diária para planejar uma assistência em saúde com base nas peculiaridades da população e que possam fundamentar ações de promoção da saúde eficazes.

Desse modo, o presente estudo tem por objetivo propor diagnósticos, resultados e intervenções de enfermagem para Pessoas em Situação de Rua com base em suas atividades de vida diária.

\section{Métodos}

Estudo descritivo, transversal, de abordagem quantitativa, realizado no período de abril a junho de 2017 no Centro de Referência Especializado para Pessoas em Situação de Rua denominado como Centro POP, em Sobral, Ceará, Brasil. Trata-se de unidade pública e estatal de referência no atendimento especializado à população adulta que vive em situação de rua ${ }^{7}$.

O público-alvo do estudo foram 60 PsR que frequentavam o referido Centro POP. Os critérios de inclusão foram: ser usuário do Centro POP e possuir idade igual ou superior a 18 anos. Foram excluídas PsR que estavam sob efeito de substâncias psicotrópicas e que apresentavam comprometimento cognitivo. Após aplicação dos critérios de elegibilidade, participaram da pesquisa 52 pessoas.

A coleta dos dados ocorreu por entrevista individual realizada por uma enfermeira e duas acadêmicas de enfermagem, com duração média de 15 minutos. Esse momento foi norteado por questionário estruturado em duas etapas, a primeira com caracterização sociodemográfica dos participantes e a segunda, com questionamentos baseados nas 12 Atividades de Vida Diárias, propostas no modelo de Roper, Logan e Tierney, que são: manter o ambiente seguro, respiração, alimentação, eliminação, comunicação, higiene pessoal e do vestuário, controle da temperatura corporal, trabalhar e divertir-se, mobilidade, exprimir a sexualidade, dormir e morrer ${ }^{9}$.

Justifica-se a escolha do modelo de Roper, Logan e Tierney, uma vez que o homem realiza uma série de atividades importantes para manutenção da saúde e do bem-estar. Nas PsR essas atividades estão estreitamente relacionadas com a saúde e podem auxiliar o profissional no planejamento e condução dos cuidados básicos, de modo a contemplar dependências na realização das atividades de vida diária9.

A coleta de dados foi realizada utilizando uma abordagem holística, para a interpretação das informações obtidas através da conversa e observação. Desse modo, inicialmente, os dados coletados foram categorizados com base nas atividades de vida diária e, em seguida, os problemas presentes em cada atividade foram analisados individualmente.

Foi utilizado o referencial de Risner, que estabelece as etapas de análise, com categorização de dados e a identificação de informações incompletas ou incongruentes; e síntese, a partir do agrupamen das evidências em padrões, a comparação dos padrões com teorias, modelos, normas e conceitos, a realização de inferências ou hipóteses e a proposição relações ${ }^{10}$.

$\mathrm{Na}$ etapa de análise, as informações contidas nas respostas dos participantes foram convertidas em características definidoras e fatores relacionados. $\mathrm{Na}$ síntese, esses resultados foram agrupados com base nas Atividades de vida diária comprometidas e em seguida foram propostos Diagnósticos de Enfermagem (DE) reais ou de risco, de acordo com a taxonomia II da NANDA-I ${ }^{11}$.

Ressalta-se que foram incluídos no plano de cuidados para PsR, apenas os DE que obtiveram frequência maior que $40 \%$. Após a definição, os mesmos foram 
associados às taxonomias Nursing Outcomes Classification (NOC), a qual padroniza o nome e as definições de resultados para uso na prática, educação e pesquisa $^{12}$. E para as intervenções de enfermagem, utilizou-se a classificação Nursing Interventions Classification (NIC), que propõe uma série de atividades que o enfermeiro pode executar com o indivíduo e comunidade ${ }^{13}$.

$\mathrm{O}$ estudo recebeu parecer favorável pelo Comitê de Ética em Pesquisa (CEP) da Universidade Estadual Vale do Acaraú (UVA), $\mathrm{n}^{\circ}$ 2.083.621 de 2017. Foram respeitados os preceitos éticos quanto à participação voluntária e consentida.

\section{Resultados}

Quanto ao perfil dos participantes do estudo, $88,5 \%$ (46) eram do sexo masculino e $11,5 \%$ (6) do sexo feminino. A média de idade foi 37,62 anos com diferença de $( \pm 9,8)$ anos. Referente ao estado civil, 51, 9\% (27) eram solteiros, 25\% (13) divorciados, 13,5\% (7) casados, $7,7 \%$ (4) estavam em união estável e 1,9\% (1) era viúvo.

Eram predominantemente católicos com 50\% (26), 30,8\% (16) representavam evangélicos, $1,9 \%$ (1) era testemunha de
Jeová e 17,3\% (9) ateus. Em relação à escolaridade, $53,84 \%$ (28) possuíam 0 a 8 anos de estudo, 40,39\% (21), 9 a 11 anos, a apenas $5,77 \%$ (3) tinham mais de 12 anos de estudo.

Dentre os motivos que os levaram a viver nas ruas, $65,39 \%$ (34) foram originados por conflitos familiares, $23,07 \%$ (12) pelo uso abusivo de drogas e 11,54\% (6) pelo desemprego. Ao avaliar o tempo que se encontravam em situação de rua, houve predomínio de vivência nas ruas de até 6 meses 38,47\% (20) e acima de 4 anos $28,84 \%$ (15). Foi identificado que $91,4 \%$ (47) relataram desejo de mudar sua situação socioeconômica e sair das ruas, entretanto $91,4 \%$ (47) estavam satisfeitos com a sua condição atual.

Foram incluídos no plano de cuidado os diagnósticos que tiveram uma frequência maior que $40 \%$. No Quadro 1 foram elencados os diagnósticos de enfermagem de cada atividade de vida, os resultados esperados e propostas de intervenções.

Quadro 1 - Diagnósticos de enfermagem, resultados esperados e principais intervenções de acordo com a ligação entre NANDA, NOC e NIC, presentes em pessoas em situação de rua. Sobral - Ceará, 2017.

\begin{tabular}{|l|l|l|l|}
\hline \multicolumn{1}{|c|}{ Atividade de vida diária } & \multicolumn{1}{|c|}{$\begin{array}{c}\text { Diagnóstico de } \\
\text { enfermagem }\end{array}$} & Resultados esperados & \multicolumn{1}{c|}{ Principais intervenções } \\
\hline $\begin{array}{l}\text { Manutenção de um ambiente } \\
\text { seguro }\end{array}$ & $\begin{array}{l}\text { Risco de dignidade } \\
\text { humana comprometida } \\
(00174)\end{array}$ & $\begin{array}{l}\text { Comportamento de } \\
\text { segurança pessoal }\end{array}$ & $\begin{array}{l}\text {-Prevenção contra violência; } \\
\text {-Prevenção no uso de drogas; } \\
\text {-Controle de impulsos; } \\
\text {-Identificação de risco. }\end{array}$ \\
\hline Comunicar & $\begin{array}{l}\text { Disposição para } \\
\text { comunicação melhorada } \\
(00157)\end{array}$ & $\begin{array}{l}\text { Capacidade de expressar } \\
\text { mensagens verbais e não } \\
\text { verbais }\end{array}$ & $\begin{array}{l}\text {-Aumento da socialização; } \\
\text {-Escutar ativamente; } \\
\text {-Promoção da integralidade } \\
\text { familiar. }\end{array}$ \\
\cline { 2 - 4 } & $\begin{array}{l}\text { Risco de vínculo } \\
\text { prejudicado (00058) }\end{array}$ & Desempenho do papel & $\begin{array}{l}\text {-Melhora na autopercepção; } \\
\text {-Promoção de vínculo; } \\
\text {-Suporte emocional; } \\
\text {-Melhora do sistema de apoio. }\end{array}$ \\
\hline Respirar & $\begin{array}{l}\text { Desobstrução ineficaz das } \\
\text { vias aéreas (00031) }\end{array}$ & $\begin{array}{l}\text { Estado respiratório: } \\
\text { Permeabilidade das vias } \\
\text { aéreas }\end{array}$ & $\begin{array}{l}\text {-Estimulação à tosse; } \\
\text {-Assistência para parar de } \\
\text { fumar; } \\
\text {-Controle da asma; } \\
\text {-Controle de infecções. }\end{array}$ \\
\hline
\end{tabular}




\begin{tabular}{|c|c|c|c|}
\hline \multirow[t]{2}{*}{ Alimentar } & $\begin{array}{l}\text { Nutrição desequilibrada: } \\
\text { menor que as necessidades } \\
\text { corporais }(00002)\end{array}$ & Autocuidado: Alimentação & $\begin{array}{l}\text {-Controle de hiperglicemia; } \\
\text {-Aconselhamento nutricional; } \\
\text {-Planejamento da dieta. }\end{array}$ \\
\hline & $\begin{array}{l}\text { Risco de contaminação } \\
(00180)\end{array}$ & $\begin{array}{l}\text { Controle de riscos e } \\
\text { conhecimento: recursos de } \\
\text { saúde }\end{array}$ & $\begin{array}{l}\text {-Identificação do risco; } \\
\text {-Controle de imunização } \\
\text {-Educação em saúde; }\end{array}$ \\
\hline \multirow[t]{2}{*}{ Eliminar } & $\begin{array}{l}\text { Eliminação urinária } \\
\text { prejudicada }(00016)\end{array}$ & Eliminação urinária & $\begin{array}{l}\text {-Assistência no autocuidado; } \\
\text {-Treinamento do hábito } \\
\text { urinário; } \\
\text {-Exercícios para a musculatura } \\
\text { pélvica; } \\
\text {-Cuidado na incontinência } \\
\text { urinária. }\end{array}$ \\
\hline & Constipação (00011) & Eliminação intestinal & $\begin{array}{l}\text {--Controle da constipação; } \\
\text {-Controle hídrico; } \\
\text {-Controle intestinal; } \\
\text {-Promoção do exercício. }\end{array}$ \\
\hline \multirow[t]{3}{*}{ Trabalho e Distração } & $\begin{array}{l}\text { Controle de impulsos } \\
\text { ineficaz (00222) }\end{array}$ & $\begin{array}{l}\text { Enfrentamento, autoestima e } \\
\text { interação social }\end{array}$ & $\begin{array}{l}\text {-Apoio emocional; } \\
\text {-Auxiliar a solucionar } \\
\text { problemas; } \\
\text {-Aconselhamento; } \\
\text {-Estimular a autoavaliação do } \\
\text { comportamento. }\end{array}$ \\
\hline & $\begin{array}{l}\text { Interação social } \\
\text { prejudicada }(00052)\end{array}$ & $\begin{array}{l}\text { Envolvimento social e } \\
\text { habilidades de interação } \\
\text { social }\end{array}$ & $\begin{array}{l}\text {-Aconselhamento; } \\
\text {-Discutir sentimentos; } \\
\text {-Terapia em grupos; } \\
\text {-Ajudar as defesas saudáveis. }\end{array}$ \\
\hline & $\begin{array}{l}\text { Comportamento de saúde } \\
\text { propenso a risco (00188) }\end{array}$ & $\begin{array}{l}\text { Controle dos sintomas, } \\
\text { comportamento de adesão }\end{array}$ & $\begin{array}{l}\text {-Estabelecimento de metas } \\
\text { mútuas; } \\
\text {-Apoio à tomada de decisão; } \\
\text {-Autorresponsabilidade. }\end{array}$ \\
\hline Sexualidade & $\begin{array}{l}\text { Padrão de sexualidade } \\
\text { ineficaz }(00065)\end{array}$ & Desempenho do papel & $\begin{array}{l}\text {-Aconselhamento sexual; } \\
\text {-Planejamento familiar; } \\
\text {-Melhora da autopercepção; } \\
\text { - Ensino: sexo seguro e melhora } \\
\text { do papel. }\end{array}$ \\
\hline \multirow[t]{2}{*}{ Sono } & $\begin{array}{l}\text { Padrão de sono } \\
\text { prejudicado }(00198)\end{array}$ & Sono & $\begin{array}{l}\text {-Aumento da segurança; } \\
\text {-Controle do ambiente; } \\
\text {-Técnica para acalmar; } \\
\text {-Terapia simples de } \\
\text { relaxamento. }\end{array}$ \\
\hline & $\begin{array}{l}\text { Conforto prejudicado } \\
(00214)\end{array}$ & Melhora do sono & $\begin{array}{l}\text {-Controle do ambiente; } \\
\text {-Facilitação do processo de } \\
\text { relaxamento; } \\
\text {-Supervisão: segurança; } \\
\text {-Relaxamento muscular. }\end{array}$ \\
\hline \multirow[t]{2}{*}{ Morte } & $\begin{array}{l}\text { Ansiedade relacionada à } \\
\text { morte }(00147)\end{array}$ & Autocontrole do medo & $\begin{array}{l}\text {-Melhora do enfrentamento; } \\
\text {-Promoção de esperança; } \\
\text {-Apoio espiritual; } \\
\text {-Redução da ansiedade; } \\
\text {-Escutar ativamente. }\end{array}$ \\
\hline & Pesar (00136) & Resolução do pesar & $\begin{array}{l}\text {-Aconselhamento; } \\
\text {-Escutar ativamente; } \\
\text {-Suporte emocional; } \\
\text {-Melhora do enfrentamento; } \\
\text {-Promoção da esperança. }\end{array}$ \\
\hline
\end{tabular}


Foram identificados $34 \mathrm{DE}$, os quais foram divididos conforme as atividades de vida diária, sendo 16 de risco e 18 reais. Os diagnósticos de risco mais prevalentes foram: comportamento de saúde propenso a risco, risco de dignidade humana comprometida, risco de vínculo prejudicado e risco de contaminação.

Já os diagnósticos reais foram: ansiedade relacionada à morte, constipação, eliminação urinária prejudicada, conforto prejudicado, pesar, padrão de sono prejudicado, interação social prejudicada, nutrição desiquilibrada: menos que as necessidades corporais, padrão de sexualidade ineficaz, controle de impulsos ineficaz, desobstrução ineficaz das vias aéreas e disposição para comunicação melhorada.

O plano de cuidados voltados à população em situação de rua foi resultante da análise das principais necessidades humanas básicas afetadas e do grau de dependência do indivíduo. Além do mais, faz-se necessário que a enfermagem, por meio da sua experiência e prática, priorize as reais necessidades do indivíduo.

\section{Discussões}

O perfil dos participantes do estudo caracteriza-se por homens, solteiros, adultos jovens e baixo nível instrucional. Tal perfil se assemelha com outros estudos realizados no Sul, Sudeste e Nordeste brasileiro ${ }^{14-16,1}$ e em outros países da América do Norte, África, Europa e América Latina ${ }^{17-29,8}$.

Por representar o maior quantitativo de PsR, indivíduos do sexo masculino devem encontrar dentro do Sistema Único de Saúde (SUS), o fortalecimento da Política Nacional para Pessoa em Situação de Rua instituída pelo Decreto ${ }^{\circ} 7.053$, de 23 de dezembro de 2009, que possa subsidiar ações que contemplem suas necessidades de saúde durante todo o seu ciclo vital.
Dos motivos relacionados com a vivência nas ruas, os conflitos familiares foram predominantes $65,39 \%$, seguido pelo uso abusivo de drogas 23,07\%. Estudo realizado com PsR de Minas Gerais identificou que $83,3 \%$ elencaram conflito e desapoio na vivência familiar como motivo para moradia na rua ${ }^{20}$. As drogas também são determinantes para conflitos intrafamiliares e se convertam também em um dos motivos para a situação de rua ${ }^{21}$. Tal problema está associado a uma série de conflitos, como precarização ou rompimento total das relações de trabalho formal e a ruptura dos vínculos familiares e comunitários $^{22}$.

Desse modo, PsR necessitam de cuidados e medidas que atendam suas particularidades. A Enfermagem vivencia em seu processo de trabalho as necessidades de saúde e isso requer estratégias de planejamento e organização, dando ênfase aos profissionais que exercem o cuidado para pessoas em situação de rua, visto as peculiaridades de cada indivíduo e os diversos fatores biopsicossociais que $o$ englobam. Sendo assim, os enfermeiros são essenciais para realizar o acompanhamento longitudinal desses indivíduos, visando garantir o acesso aos serviços de saúde e uma assistência de qualidade ${ }^{23}$.

No presente estudo houve identificação de 34 DE conforme as atividades de vida diária, sendo 16 de risco e 18 reais. Houve maior prevalência dos diagnósticos de risco comportamento de saúde propenso a risco e o risco de dignidade humana comprometida. Estudo realizado com PsR de São Paulo identificou que $80 \%$ tinham comportamentos que impediam a manutenção eficaz da saúde. Há ainda, um direcionamento deficitário das políticas públicas na promoção da saúde dessa parcela da população e o atendimento é comumente precário, seja pelo preconceito do profissional de saúde, que encontra dificuldade em se relacionar com 
os moradores de rua, seja pelo tratamento proposto $^{24}$.

Esses indivíduos têm dificuldades em adentrar nos serviços de saúde e educação devido à precariedade de sua sobrevivência, além da violência que estão expostos diariamente, tornando-os mais susceptíveis às doenças e agravos. Portanto, os profissionais de saúde devem acolher esse público, prestando cuidado qualificado e sendo educadores, orientando-os para que os mesmos sejam capazes de realizar autocuidado e estreitem laços com os serviços de saúde ${ }^{15}$.

Constantemente, o risco de dignidade humana também é comprometido devido à violência que estão expostos nas ruas, já que a maioria não dispõe de lugar fixo e seguro para dormir, acarretando outros diagnósticos, padrão de sono e conforto prejudicados ${ }^{3}$.

Pode-se observar o risco de vínculo prejudicado, encontrado também em outro estudo realizado no Rio Grande do Norte e que traz como principal motivo para distanciá-los da família e do convívio social, o uso abusivo de álcool, consequentemente ocasionando interação social prejudicada ${ }^{6}$.

A perda de vínculo social trata-se de processo gradual e agudo de desvinculação social e que a PsR se encontra no centro do limiar entre a pobreza e a miséria. Diante disso, por vezes se usam como estratégias de sobrevivência pedir esmolas ou se envolver com atividades ilícitas, o que corrobora para a construção de identidade social marginalizada ${ }^{25}$.

Também foram identificados diagnósticos: desobstrução ineficaz das vias aéreas, risco de contaminação, eliminação urinária prejudicada, constipação, controle de impulsos ineficaz, padrão de sexualidade ineficaz, ansiedade relacionada à morte e pesar, gerando prejuízos na qualidade de vida desses indivíduos. Foi possível notar que um diagnóstico acarreta outro e que os mesmos são interligados.

Os diagnósticos relacionados à respiração são reflexos da exposição constante ao ambiente hostil das ruas, ao frio, vento forte e chuva. As doenças respiratórias podem ser agravadas devido às iniquidades resultantes da inserção desigual dos indivíduos na sociedade. Além de ser um risco para tuberculose, a situação de rua representa também um aumento de desfechos negativos da doença ${ }^{26}$.

Pesquisa realizada em Minas gerais com 20 PsR corrobora com os achados desse estudo, confirmando a presença de problemas emocionais, de eliminação e sexualidade. A dificuldade para atender às necessidades básicas é uma das principais causas para tais problemas, logo, garantir o acesso à higiene e cuidados pessoais pode fortalecer a autoestima e reduzir o preconceito $^{26}$.

Os principais resultados esperados de acordo com outro estudo consultado foram: autocuidado, controle de riscos, envolvimento social e comportamento de adesão. Já as intervenções de enfermagem mais relevantes foram: assistência no autocuidado, aconselhamento, controle de impulsos, identificação de risco, planejamento da dieta, terapia em grupos, promoção da esperança, escutar ativamente, ensino, educação em saúde, redução da ansiedade e apoio espiritual ${ }^{6}$.

As intervenções de enfermagem são importantes para inserir esses indivíduos não só nos serviços de saúde como na sociedade, melhorando a socialização e comunicação, através do acolhimento apropriado aos usuários, da escuta ativa e do apoio, para que os mesmos retomem a esperança na dignidade humana ${ }^{6}$.

Destaca-se que o planejamento de intervenções emancipatórias constitui elemento importante, pois traz o sujeito para o centro do cuidado, delimitando sua identidade e necessidades para a construção das práticas de saúde. Assim, incluir o sujeito no centro do cuidado representa colocá-lo como protagonista de sua própria vida $^{27}$.

Além disso, as intervenções de enfermagem devem ser focadas em um sistema de apoio e educação em saúde para 
estimular a realização adequada das atividades de vida diária. Identificar as necessidades de saúde dessa população por meio do processo de enfermagem pode estabelecer os elos necessários à implementação das ações de enfermagem. A PsR requer atendimento multidisciplinar e intersetorial e precisa ser vista à luz da equidade e universalidade garantida pelos princípios do $\mathrm{SUS}^{8}$.

Nesse contexto, ressalta-se que a sistematização do cuidado é um instrumento que fortalece a tomada de decisões, com respeito às diferenças, potencializando as capacidades individuais, sociais e coletivas, considerando o contexto real em que as pessoas vivem, possibilitando a realização do cuidado humano de forma holística e segura adequando as reais necessidades de cada indivíduo ${ }^{28}$

Portanto, espera-se que os achados desse estudo possam contribuir para reflexões acerca das necessidades de intervenções voltadas para um cuidado efetivo com essa população e que a prática da enfermagem seja cada vez mais humanizada, respeitando as particularidades desse grupo que, na maioria das vezes, é excluído. Destaca-se que as intervenções não foram implementadas, o estudo consumou-se até a etapa de planejamento (plano de cuidados).

As limitações relacionam-se com a operacionalização da coleta de dados, devido às peculiaridades do cenário de pesquisa. $\mathrm{O}$ fato de ter sido realizado com PsR acompanhadas por um Centro POP, também configura limitação, pois as informações coletadas podem divergir de indivíduos sem esse apoio.

Ressalta-se que mais estudos com uma equipe multidisciplinar são necessários a fim de implementar o plano de cuidados construído neste estudo e, também, para obter uma melhor compreensão das potencialidades de dificuldades encontradas nesse processo. Além disso, os estudos são importantes para aumentar a visibilidade desse grupo diante de gestores públicos, pesquisadores e população em geral.

\section{Conclusão}

O estudo apresentou perfil de pessoas em situação de rua majoritariamente do sexo masculino, adultos jovens, solteiros e com pouco grau de instrução. Foi possível identificar por meio das atividades de vida diária, 34 diagnósticos reais e potenciais. Os achados reforçam a importância da padronização do plano de cuidados, formado por diagnósticos de enfermagem, resultados esperados e intervenções, como forma de esclarecer as necessidades de saúde da população de rua e, consequentemente, evidenciar a importância da atuação do enfermeiro na equipe multiprofissional.

Dentro da SAE, os diagnósticos de enfermagem podem permitir a identificação de intervenções necessárias para desenvolver resultados de responsabilidade do profissional enfermeiro e efetuar a assistência de enfermagem com base nos diversos problemas que envolvem $\mathrm{o}$ processo saúde-doença da PsR.

Apesar de o estudo apresentar limitação do público, por englobar apenas PsR frequentadoras do Centro POP, o mesmo apresentou a relevância da identificação dos diagnósticos, além da elaboração do plano de cuidados, reflexão das problemáticas e necessidades das PsR, longe de preconceitos e negligências. Vale salientar ainda a escassez de estudos sobre a temática, dessa forma, torna-se imperativa a realização de novos estudos sobre SAE direcionados a esta população.

\section{Referências}

1. Bezerra IHP, Macêdo Filho I, Costa RJLM, Sousa VJ, Carvalho MVG. População em situação de rua: um olhar da enfermagem sobre o processo saúde/doença. Enferm 
Revista. [Internet] 2015 [citado 2019 abril 23]; 18(1):3-14. Disponível em: http://periodicos.pucminas.br/index.php/enfermagemrevista/article/view/9365

2. Fazel S, Geddes JR, Kushel M. The health of homeless peopa terle in high-income countries: descriptive epidemiology, health consequences, and clinical and policy recommendations. Lancet. [Internet] 2014 [cited 2019 april 23]; 384(9953):1529-1540. Available from: https:/www.ncbi.nlm.nih.gov/pmc/articles/PMC4520328/

3. Rae BE, Rees S. The perceptions of homeless people regarding their healthcare needs and experiences of receiving health care. J Adv Nurs. [Internet] 2015 [cited 2019 april 23]; 71:2096-107. Available from: https://www.ncbi.nlm.nih.gov/pubmed/25916241

4. Feral-Pierssens AL, Aubry A, Truchot J, Raynal PA, Boiffier M, Hutin A, et al. Emergency Care for Homeless Patients: A French Multicenter Cohort Study. Am J Public Health. [Internet] 2016; [cited 2019 april 23]; 106(5):893-8. Available from: https://www.ncbi.nlm.nih.gov/pubmed/26985613

5. Londero MFP, Ceccim RB, Bilibio LFS. Consultório de/na rua: desafio para um cuidado em verso na saúde. Interface Comun Saúde Educ. [Internet] 2014 [citado 2019 abril 23]; 18: 251-60. Disponível em: http://www.scielo.br/scielo.php?pid=S141432832014000200251\&script $=$ sci abstract\&tlng $=$ pt

6. Teixeira GA, Carvalho JBL, Silva ALMAD, Santos SBD, Lopes TRG. Sistematização da assistência de enfermagem a pessoa em situação de rua. Journal of Nursing UFPE. [Internet] 2015 [citado 2019 abril 20]; 9(3):7169-7174. Disponível em: https://periodicos.ufpe.br/revistas/revistaenfermagem/article/view/10448/11263

7. Santos WN. Systematization of nursing care: the historical context, the process and obstacles to deployment. J Manag Prim Health Care. 2014; 5(2):153-158.

8. da Silva FP, de Assis Siqueira Paiva F, Guedes CP, da Silva Frazão I, Vasconcelos SC, da Costa Lima MD. Nursing Diagnoses of the Homeless Population in Light of Selfcare Theory. Arch Psychiatr Nurs. [Internet] 2018 [cited 2021 January 02];32(3):425431. Available from: http://dx.doi.org/10.1016/j.apnu.2017.12.009

9. Roper N, Logan W, Tierney A. The Roper, Logan, Tierney Modelo of Nursing based on activities of living. London: Churchill Livingstone, 2000.

10. Risner PB. Diagnosis: analysis and synthesis of data. In: Griffith-Kenney JW, Christensen PJ. Nursing Process application of theories, frameworks, and models. 2. ed. St. Louis, Mosby. 1986

11. Nanda Internacional. Diagnósticos de enfermagem da NANDA: definições e classificação 2015-2017. Porto Alegre: Artmed; 2015.

12. Moorhead S, Johnson M, Maas M, Swanson E. Classificação dos Resultados de Enfermagem: mensuração dos resultados em saúde. $5^{\text {a }}$ ed. Rio de Janeiro: Elsevier; 2016.

13. Bulechek GM, Butcher HK, Dochterman JM, Wagner CM. Classificação das intervenções de enfermagem (NIC). $6^{\mathrm{a}}$ ed. Rio de Janeiro: Elsevier; 2016.

14. Lussi IAO, Ricci TE, Pinho RJ. Perceptions on work: analysis of conceptions of homeless people. Cad. Bras. Ter. Ocup. [Internet] 2017 [cited 2019 april 20]; 25(4): 779-793.

Available from:http://www.cadernosdeterapiaocupacional.ufscar.br/index.php/cadernos/article/vi ewFile/1677/912

15. Cunha JG, Garcia A, Silva THS, Pinho RC. Novos arranjos: lançando um olhar sobre os relacionamentos interpessoais de pessoas em situação de rua. Rev Interinstitucional de Psicologia [Internet] 2017 [citado 2019 abril 23]; 10 (1): 95 - 108. Disponível em: http://pepsic.bvsalud.org/pdf/gerais/v10n1/10.pdf 
16. Moura Jr JF, Ximenes VM. A identidade social estigmatizada de pobre: uma constituição opressora. Fractal: Rev de Psicologia [Internet] 2016 [citado 2019 abril 20]; 28(1): 76-83. Disponível em: http://dx.doi.org/10.1590/1984-0292/1051

17. Stajduhar KI, Mollison A, Giesbrecht M, McNeil R, Pauly B, Reimer-Kirkham S, et al. "Just too busy living in the moment and surviving": barriers to accessing health care for structurally vulnerable populations at end-of-life. BMC Palliat Care. [Internet] 2019 [cited 2019 april 19]; 18 (1):11-20. Available from: https://www.ncbi.nlm.nih.gov/pubmed/30684959

18. Tinland A, Boyer L, Loubière S, Greacen T, Girard V, Boucekine $\mathrm{M}$, et al. Victimization and posttraumatic stress disorder in homeless women with mental illness are associated with depression, suicide, and quality of life. Neuropsychiatr Dis Treat. [Internet] 2018 [cited 2019 april 19]; (4) 14: 2269-2279. Available from: https://www.dovepress.com/victimization-and-posttraumatic-stress-disorderhomeless-women-with-peer-reviewed-article-NDT

19. Ayano G, Assefa D, Haile K, Chaka A, Solomon H, Hagos P, et al. Mental, neurologic, and substance use (MNS) disorders among street homeless people in Ethiopia. Annals of General Psychiatry, [Internet] 2017 [cited 2019 april 23]; 16:40. Available from: https://www.ncbi.nlm.nih.gov/pubmed/29176996

20. Balieiro EO, Soares CPD, Vieira EA. Morador de rua: causas, entraves e serviços ofertados. Temporalis [Internet] 2017 [citado 2019 abril 23]; 17(34): 333-353. Disponível em: http://periodicos.ufes.br/temporalis/article/view/14606/0

21. Caravaca MJA, Padilha MI. A dinâmica das relações familiares de moradores de rua usuários de crack. Saúde debate [Internet]. 2015 Sep [citado 2019 abril 23] ; 39( 106 ): 748-759. Disponível em: http://www.scielo.br/scielo.php?script=sci_arttext\&pid=S010311042015000300748\&1 $\underline{\text { ng}}=$ en.

22. Brandão MGSA, Ximenes MAM, Aragão CP, Caetano JÁ, Araújo TM, Barros LM. Autoconceito, depressão e ansiedade de pessoas em situação de rua. Revista Enfermagem Atual In Derme [Internet]. 2019 [citado 2020 abril 23]; 90(28): 1-6. Disponível em: https://revistaenfermagematual.com/index.php/revista/article/view/404/554

23. Cardoso AC, Santos DS, Mishima SM, Anjos DSC, Jorge JS, Santana HP. Challenges and potentialities of nursing work in street medical offices. Rev. Latino-Am. Enfermagem. [Internet] 2018 [cited 2019 april 20]; 26:e3045. Available from: http://www.revenf.bvs.br/pdf/rlae/v26/pt_0104-1169-rlae-26-e3045.pdf.

24. Tiradentes LR, Fernandes RAQ. Nursing diagnoses identified in homeless women. Online Brazilian Journal of Nursing [Internet] 2007 [cited 2019 april 19]; 7(1): 1-11. Available from: http://www.objnursing.uff.br/index.php/nursing/article/view/j.16764285.2008.1356/431.

25. Medeiros FC, Matos ACV, Pagnussat E, Oliveira IMFF. Entre a benesse e o direito: as políticas de atendimento à população em situação de rua na América Latina. Psicol. Estud. [Internet] 2020 [citado 2021 janeiro 05]; 25: e45025. Disponível em: http://www.scielo.br/scielo.php?script=sci_arttext\&pid=S141373722020000100237\&1 ng=en\&nrm=iso

26. 26. Valle FAAL, Farah BF, Carneiro JN. As vivências na rua que interferem na saúde: perspectiva da população em situação de rua. Saúde debate [Internet]. 2020 Mar [citado 2021 janeiro 05]; 44(124): 182-192. Disponível em: http://www.scielo.br/scielo.php?script=sci_arttext\&pid=S010311042020000100182\&1 $\underline{\text { ng}}=$ en 
27. Koopmans FF, Daher DV, Acioli S, Sabóia VM, Ribeiro CRB, Silva CSSL. O viver na rua: Revisão integrativa sobre cuidados a moradores de rua. Rev. Bras. Enferm. [Internet]. 2019 [citado 2021 janeiro 05]; 72(1): 211-220. Disponível em: http://www.scielo.br/scielo.php?script=sci arttext\&pid=S003471672019000100211\&1 $\underline{\text { ng=en }}$

28. Hoffelder GK, Vicensi MC. Caracterização dos diagnósticos de enfermagem como instrumento do cuidado humano. Unoesc \& Ciência - ACBS; 2014; 5(2):135-142.

Como citar este artigo:

Ximenes AM, Muniz IF, Caetano JÁ, Brandão MGSA, Aragão CP, Barros LM. Proposta de plano de cuidados para pessoas em situação de rua. Rev. Aten. Saúde. 2021; 19(67): 154-164. 